\title{
Is PCSK9 Associated with Plasma Lipid Levels in a Sub-Saharan African Population of Patients with Obesity and Type 2 Diabetes?
}

This article was published in the following Dove Press journal: Diabetes, Metabolic Syndrome and Obesity: Targets and Therapy

\author{
Camille Maadjhou Mba $\mathbb{D}^{\prime}$ \\ Wilfred Mbacham' \\ Eugène Sobngwi ${ }^{2,3}$ \\ Jean Claude Mbanya ${ }^{2,3}$
}

'Department of Physiological Sciences/ Biochemistry, Faculty of Medicine and Biomedical Sciences, University of Yaoundé I, Yaoundé, Cameroon; ${ }^{2}$ Department of Internal Medicine and Specialties, Faculty of Medicine and Biomedical Sciences, University of Yaoundé I, Yaoundé, Cameroon; ${ }^{3}$ National Obesity Center and Endocrine and Metabolic Diseases Unit, Yaoundé Central Hospital, Yaoundé, Cameroon

Correspondence: Camille Maadjhou Mba Department of Physiological Sciences/ Biochemistry, Faculty of Medicine and Biomedical Sciences, University of Yaoundé I, PO Box 1364, Yaoundé, Cameroon

Email camilya91@yahoo.fr

\begin{abstract}
Purpose: Proprotein convertase subtilisin/kexin type 9 (PCSK9) is a key regulator of circulating LDL cholesterol. There are inconsistent data in some populations concerning the association between PCSK9, LDL and CRP. The emerging importance of the inhibition of PCSK9 for the treatment of hypercholesterolemia warrants investigations in different populations. The aim of this study from a Sub-Saharan African population was to evaluate the association between PCSK9 and hs-CRP levels and plasma lipid levels in patients with type 2 diabetes (T2D) and obese and lean controls.
\end{abstract}

Patients and methods: A cross-sectional analytical study was conducted in a major hospital in Yaoundé, Cameroon in a cohort of 162 participants (53\% females). There were 54 non-obese T2D patients matched for age and sex to 54 obese nondiabetic and 54 nondiabetic lean subjects. PCSK9 level was assessed by sandwich ELISA method and hsCRP by nephelometry.

Results: PCSK9 and hs-CRP levels were significantly higher in obese and T2D subjects when compared to lean controls $(\mathrm{p}<0.001$ and $\mathrm{p}=0.002$, respectively). The association between PCSK9 and triglyceride levels in the overall population was gender dependent $(\mathrm{p}=0.04)$ and subgroup analysis showed a significant positive correlation between PCSK9 and triglyceride levels in males but not in females $(r=0.56, \mathrm{p}=0.02$ and $r=0.2$ and $\mathrm{p}=0.1$, respectively). Multilinear regression analysis identified BMI as an independent predictor for PCSK9 levels and this association was maintained after adjustment for confounders; adjusted $\beta$-coefficient; 36.1 (95\% CI; 29.2-47.4). We did not find an association between PCSK9 and any plasma lipid levels in obese and T2D subjects, nor between PCSK9 and hs-CRP levels. Conclusion: Obese and type 2 diabetes subjects have higher PCSK9 levels when compared to lean controls, suggesting that these metabolic states potentially impact PCSK9 levels in Cameroonian patients.

Keywords: PCSK9, LDL-cholesterol, type 2 diabetes, obesity

\section{Introduction}

The global prevalence of type 2 diabetes (T2D) is rising possibly due to obesity, changing lifestyle and physical inactivity. ${ }^{1} \mathrm{~T} 2 \mathrm{D}$ and obesity are independent cardiovascular risk factors. An important contributor to elevated cardiovascular disease (CVD) risk in T2D and obese subjects is dyslipidemia including abnormalities in all lipoproteins. T2D and obesity are associated with increased levels of small dense low-density lipoprotein cholesterol (LDL), decreased levels of high-density lipoprotein (HDL) cholesterol, and increased levels of triglycerides. ${ }^{2,3}$ 
The International Diabetes Federation (IDF) recommends a triple target achievement (glycated hemoglobin $<7 \%$, blood pressure $<130 / 80 \mathrm{mmHg}$ and LDL cholesterol $<100 \mathrm{mg} / \mathrm{dL}$ ) in patients with diabetes. In Africa, the number of patients achieving the triple targets is low. ${ }^{1}$ Management of diabetic dyslipidemia is essential in the multifactorial approach to prevent CVD in patients with $\mathrm{T} 2 \mathrm{D} .{ }^{4}$ One of the key regulators of circulating LDL is proprotein convertase subtilisin/kexin type 9 (PCSK9). It promotes the lysosomal degradation of hepatic low-density lipoprotein receptors, resulting in increased circulating LDL cholesterol levels and therefore regarded as a good lowering lipid target. ${ }^{5,6}$ Moreover, observational studies suggest a weak positive correlation between PCSK9 and CRP, but a recent review revealed that PCSK9 inhibitors do not alter hs-CRP levels. ${ }^{6,7}$

There are inconsistent findings in the association between PCSK9 measured in ethnically diverse cohorts and plasma LDL concentrations. Some studies found PCSK9 level elevated in patients with obesity and T2D and others not. ${ }^{8,9}$ Studies have reported that insulin induces PCSK9 expression, suggesting that insulinresistant states like T2D and obesity may drive circulating PCSK9 levels. ${ }^{10,11}$

To date, there is limited data on PCSK9 concentrations in the African population and evidence supporting its association with circulating LDL cholesterol levels is unclear. $^{12,13}$ Studies largely conducted in Caucasian and Asian populations suggest that PCSK9 levels are increased, unchanged or oven reduced in subjects with obesity and T2D. ${ }^{8,9,14}$ It is however unclear whether PCSK9 levels are also changed in African adults with type 2 diabetes and obesity. The purpose of this study in a sub-Saharan African population was to investigate the association between PCSK9 and plasma lipid levels in adults with obesity and T2D.

\section{Methods}

\section{Study Design and Study Population}

We conducted a cross-sectional analytical study at a major hospital in Yaoundé, Cameroon. The study protocol was in accordance with the principles of the Declaration of Helsinki and approved by the Centre Regional Ethics Committee for Human Health Research, Cameroon. All participants provided written informed consent prior to inclusion.
Participants were patients with obesity without T2D and non-obese T2D patients recruited by systematic sampling from the major diabetes center in the country and matched for age and sex to lean subjects without diabetes. $\mathrm{T} 2 \mathrm{D}$ was defined as fasting plasma glucose $\geq 1.26 \mathrm{~g} / \mathrm{L}$ on 2 separate occasions or as documented diagnosis and/or intake of anti-diabetic medication; obesity as body mass index $(\mathrm{BMI}) \geq 30 \mathrm{Kg} / \mathrm{m}^{2}$; and lean subjects as BMI $<25 \mathrm{Kg} /$ $\mathrm{m}^{2}$. Non-diabetes were people without history of diabetes and who had $\mathrm{HbAl}_{\mathrm{C}}<6.5 \%$. Participants were not eligible if pregnant, on lipid-lowering medication or refused to give informed written consent.

Sample size calculation was done using Whitley and Ball's formula which is one used for calculation for a difference in means between 2 or more groups. ${ }^{15}$ To obtain a mean difference of $45 \mathrm{ng} / \mathrm{mL}$ of PCSK9 levels between the groups, we estimated a sample size of 52 patients per group from $80 \%$ power and $5 \%$ alpha.

\section{Data Collection}

Demographics, medical history, lifestyle and anthropometrics were obtained during an in-person visit completed by all participants. Clinical examination measured blood pressure, weight and height twice and an average of the two measures considered. Trained investigators measured weight in participants without shoes, belt or jackets using an electronic scale and height in participants without shoes using a portable stadiometer that was calibrated prior to use. Body mass index was calculated as weight in kilograms divided by the square of height in meters. Blood pressure was measured in a sitting position after at least $10 \mathrm{~min}$ of rest using an automatic sphygmomanometer $\left(\mathrm{OMRON}^{\circledR}\right.$ HEM712 C, OMRON HEALTHCARE, INC. Bannockburn, Illinois 60015. USA). We defined hypertension as systolic blood pressure (SBP) $\geq 140 \mathrm{mmHg}$ and/or diastolic blood pressure (DBP) $\geq 90 \mathrm{mmHg}$, or antihypertensive drug use.

Fasting venous blood samples were collected from participants (after at least $10 \mathrm{hrs}$ of overnight fast) for laboratory analysis in 3 different tubes: dry, EDTA and sodium fluoride tubes. Plasma and serum were separated and aliquoted within $30 \mathrm{~min}$ of collection and stored at $-80^{\circ} \mathrm{C}$ until analysis. Serum triglycerides (TG) and highdensity lipoprotein (HDL)-cholesterol and total cholesterol (TC) were measured by end-point colorimetric method using Biolabo ${ }^{\mathrm{TM}}$ kits. The inter-assay coefficients of variation were $2.5 \%$ and $1.8 \%$ and $1.8 \%$, respectively. Lowdensity lipoproteins (LDL) cholesterol concentration was 
calculated using the Friedewald equation: LDL $(\mathrm{g} / \mathrm{L})=\mathrm{TC}$ $(\mathrm{g} / \mathrm{L})-\mathrm{HDL}(\mathrm{g} / \mathrm{L})-\mathrm{TG}(\mathrm{g} / \mathrm{L}) / 5$, provided $\mathrm{TG}$ is $<4 \mathrm{~g} / \mathrm{L}$.

$\mathrm{HbAlc}$ was assayed by the boronate affinity chromatography methods using LabonaCheck ${ }^{\mathrm{TM}}$ A1c. Fasting glycaemia was measured by enzymatic method using the highly specific enzyme glucose oxidase. Plasma levels of PCSK9 were measured using a commercial high-sensitivity, quantitative sandwich enzyme immunoassay (Human PCSK9 PicoKine ${ }^{\mathrm{TM}}$ ELISA Kit Boster Biological Technology, Pleasanton CA, USA, Catalog \# EK1147) according to the manufacturer's instructions. Plasma samples were diluted 1:100 in the diluent buffer. This dilution factor had been determined as appropriate by titration assay according to the manufacturer prior to the batch measurement. The typical assay range of this ELISA kit is between $156 \mathrm{pg} / \mathrm{mL}-10000 \mathrm{pg} / \mathrm{mL}$. The intra-assay coefficient of variation was $7.6 \%$ and inter assays was $9.8 \%$. Highly sensitive C-reactive protein (hs-CRP) was assayed by high-sensitivity enzyme-linked immunosorbent assay using nephelometry method (Genrui Biotech Inc).

\section{Statistical Analysis}

All analyses were performed using IBM SPSS statistics for windows version 20.0. Armonk, NY: IBM Corp. Continuous variables are presented as median and categorical variables are expressed as frequency and percent. Differences between groups were computed using Kruskal-Wallis test for continuous variables and chi2 test for categorical variable, with Bonferroni correction for pairwise comparison $(p<0.017)$.
Correlations between clinical variables and PCSK9 were determined using Spearman correlation. Multivariate linear regression models were constructed to identify independent predictors for PCSK9 and adjustments for possible confounding factors such as age, sex, HbAlc and BMI made by including them as covariates in the multilinear linear regression model. Where applicable, a p-value $<0.05$ was considered as statistically significant.

\section{Results}

A total of 162 participants were recruited; 54 patients with T2D but without obesity, matched for age and sex to 54 patients with obesity but no diabetes and 54 nondiabetic lean subjects. Descriptive characteristics for the patients are shown in Table 1.

Participants had a median age of 54.5 years [46-61], with a median known duration of diabetes of 5.1 years [2.9-7.6] for the diabetes group. Majority of participants were females $53 \%$. Approximately $35 \%$ of T2D patients reported a prior diagnosis of high blood pressure, as compared to almost $13 \%$ in the group with obesity and $4 \%$ in the lean group. Median BMI in the group with obesity was $37.1 \mathrm{Kg} / \mathrm{m} 2$, as opposed to $24.8 \mathrm{Kg} / \mathrm{m} 2$ in the $\mathrm{T} 2 \mathrm{D}$ group and $24.1 \mathrm{Kg} / \mathrm{m} 2$ in the lean group as shown in Table 1 .

Table 2 shows that overall, fasting blood glucose increased from the lean to obese $(\mathrm{p}<0.001)$, and obese to type 2 diabetes groups $(\mathrm{p}<0.001)$. Total cholesterol, LDL cholesterol and triglycerides levels differed significantly

Table I Demographic Characteristics of Study Participants According to Groups

\begin{tabular}{|c|c|c|c|c|}
\hline Patient Characteristics & $\begin{array}{l}\text { Type } 2 \text { Diabetes (T2D) Non } \\
\text { Obese n= } 54 \text { M[QI-Q3] }\end{array}$ & $\begin{array}{l}\text { Obese Non T2D n= } 54 \text { M } \\
\text { [QI-Q3] }\end{array}$ & $\begin{array}{l}\text { Lean Non T2D n= } 54 \mathrm{M} \\
{[\mathrm{Q} /-\mathrm{Q} 3]}\end{array}$ & p-value \\
\hline Age (years) [QI-Q3] & $54.5[46.0-61.0]$ & $54.5[46.0-61.0]$ & $54.5[46.0-61.0]$ & 0.40 \\
\hline Female, n (\%) & $29(53.7)$ & $29(53.7)$ & $29(53.7)$ & 0.86 \\
\hline $\begin{array}{l}\text { Known duration of diabetes } \\
\text { (years) }\end{array}$ & $5.1[2.9-7.6]$ & - & - & - \\
\hline Known hypertension, n(\%) & $19(35.1)$ & 7 (12.9) & $2(3.7)$ & 0.01 \\
\hline \multicolumn{5}{|l|}{ Medication, n (\%): } \\
\hline Oral hypoglycemic agents & $54(100)$ & - & - & - \\
\hline Insulin & $9(18)$ & - & - & 一 \\
\hline Anti-hypertensive drugs & $16(29.6)$ & $4(7.4)$ & $2(3.7)$ & 0.006 \\
\hline $\mathrm{BMI}(\mathrm{Kg} / \mathrm{m} 2)$ & $24.8[23.1-26.3]$ & $37.1[32.4-44.38]$ & $24.1[23.7-24.6]$ & $<0.001$ \\
\hline Systolic BP (mmHg) & $\mid 26.5[|| 5.5-\mid 39.8]$ & $|3| .0[121.0-140.0]$ & $\mid 25.5[|| 5.8-\mid 28.3]$ & 0.116 \\
\hline Diastolic BP (mmHg) & $78.0[73.0-89]$ & $85.0[78.0-91.0]$ & $79.5[71.5-87.0]$ & 0.195 \\
\hline Heart rate (bpm) & $76.5[72.0-82.3]$ & $77.0[70.0-82.0]$ & $72.5[70.0-77.3]$ & 0.111 \\
\hline
\end{tabular}

Notes: Indicates data not available because it was not appropriate. Boldface type indicates statistical significance where $\mathrm{p}<0.05$. Values are given in M[QI-Q3] unless otherwise stated. - indicates data not available because it was not appropriate. Table I shows the descriptive characteristics of the study participants. There was no difference in age, sex ratio and mean blood pressure between the 3 groups. The diabetes group had a significantly higher number of patients with known high blood pressure. Abbreviations: M[QI-Q3], Median [25th-75th percentile]; T2D, type 2 diabetes; BMI, body mass index; BP, blood pressure; bpm, beats per minutes. 
Table 2 Biological Findings of Study Participants According to Groups

\begin{tabular}{|c|c|c|c|c|}
\hline Patient Characteristics & Type 2 Diabetes $n=54$ M[Q I-Q3] & Obese $n=54 M[Q 1-Q 3]$ & Lean n =54 M[QI-Q3] & p-value \\
\hline FBG $(g / L)$ & I.07[1.02-1.39] & $1.05[1.03-1.09]$ & $1.01[0.97-1.06]$ & 0.012 \\
\hline HbAlc (\%) & $6.05[4.93-6.98]$ & $5.94[4.80-6.50]$ & $5.1[4.60-5.39]$ & 0.06 \\
\hline LDL cholesterol (g/L) & $0.98[0.83-1.09]$ & $0.93[0.85-1.05]$ & $0.88[0.74-1.03]$ & 0.007 \\
\hline HDL-Cholesterol (g/L) & $0.40[0.38-0.46]$ & $0.43[0.38-0.49]$ & $0.53[0.44-0.57]$ & 0.68 \\
\hline Total cholesterol (g/L) & $1.73[1.58-1.85]$ & $1.74[1.62-1.85]$ & $1.67[1.54-1.80]$ & 0.01 \\
\hline Triglycerides (g/L) & $1.78[1.45-2.01]$ & $1.65[1.43-1.92]$ & $1.58[1.32-1.47]$ & 0.018 \\
\hline hs-CRP (mg/dL) & $1.36[0.22-3.01]$ & $\mathrm{I} .81[0.57-4.42]$ & $0.27[0.20-0.66]$ & 0.002 \\
\hline
\end{tabular}

Notes: Boldface type indicates statistical significance where $\mathrm{p}<0.05$. Patients with obesity and type 2 diabetes had significantly higher levels of PCSK9, hs-CRP, triglycerides, total cholesterol and fasting glycaemia than the lean group.

Abbreviations: M[QI-Q3], Median [25th-75th percentile]; FPG, Fasting Plasma Glucose; HbAIc, Glycated hemoglobin; HDL, high-density lipoprotein; LDL, low-density lipoprotein; hs-CRP, High sensitive C-reactive protein.

between the type 2 diabetes and lean group only $(\mathrm{p}=0.014$, $\mathrm{p}=0.009$ and $\mathrm{p}=0.012$, respectively). Mean hs-CRP levels were significantly different between the 3 groups $(p=0.002)$.

As shown in Figure 1, PCSK9 levels were significantly higher in type 2 diabetes compared to lean subjects $(\mathrm{p}=0.001)$ and in obese compared to lean subjects $(p=0.003)$. PCSK9 concentrations did not differ significantly between subjects with type 2 diabetes and those with obesity. Subgroup analyses by sex showed that in males, the mean PCSK9 concentration of the lean group was significantly different from both the type 2 diabetes group $(p=0.001)$ and the obese group $(p=0.011)$. In females, PCSK9 levels differed significantly between the groups (0.041) (Figure 2).

Table 3 shows correlation analyses of PCSK9 with different clinical variables according to gender. A significant positive correlation was found between PCSK9 levels and BMI in females and between PCSK9 and triglycerides levels

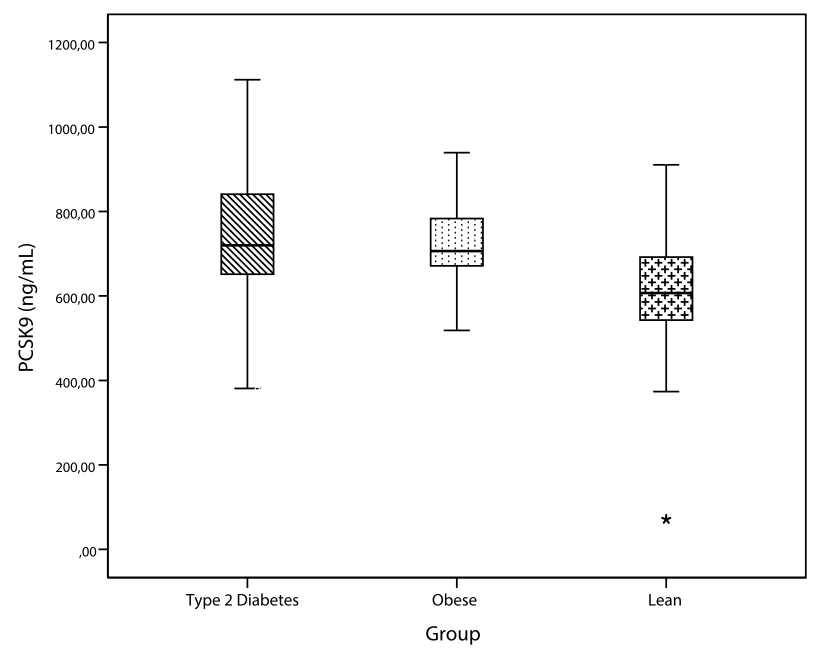

Figure I Comparison of PCSK9 levels by group. PCSK9 was significantly higher in patients with obesity and type 2 diabetes. in males. There was no association between PCSK9 and hsCRP, nor between PCSK9 levels and markers of glycemic control (HbA1C or fasting glycaemia). Correlation analyses by groups (type 2 diabetes, obese and lean groups) did not show any significant correlation between PCSK9 and the clinical or biochemical parameters.

On performing multilinear regression model, BMI was identified as an independent predictor for PCSK9 levels in the total population and this relationship was unchanged after adjusting for confounders (age, sex, HbAlc and known high blood pressure): Crude $\beta$-coefficient 42.1 (95\% CI; 33.5-52.7) vs adjusted $\beta$-coefficient 36.1 (29.2-47.4). There was no association between PCSK9 and any of the plasma lipids measured even after adjustment for the confounders listed above.

\section{Discussion}

Scanty information on proprotein convertase subtilisin kexin (PCSK9) exists in Africa. PCSK9 genetic variants cause extreme plasma LDL cholesterol (LDL-C) deviations. We sought to evaluate the relationship between PCSK9 levels and plasma lipid levels in patients with type 2 diabetes (T2D) comparatively to matched patients with obesity without diabetes and lean subjects. The major finding is that PCSK9 and hs-CRP levels were significantly different between the 3 groups. BMI was identified as an independent predictor for PCSK9 levels, and this relationship was unchanged after adjusting for potential confounders such as age, sex, blood pressure and glycated hemoglobin levels. PCSK9 levels were positively correlated with serum triglyceride levels in the male group.

Our study showed that PCSK9 levels were significantly higher in patients with obesity and type 2 diabetes and this remained unchanged on subgroup analysis by gender. 


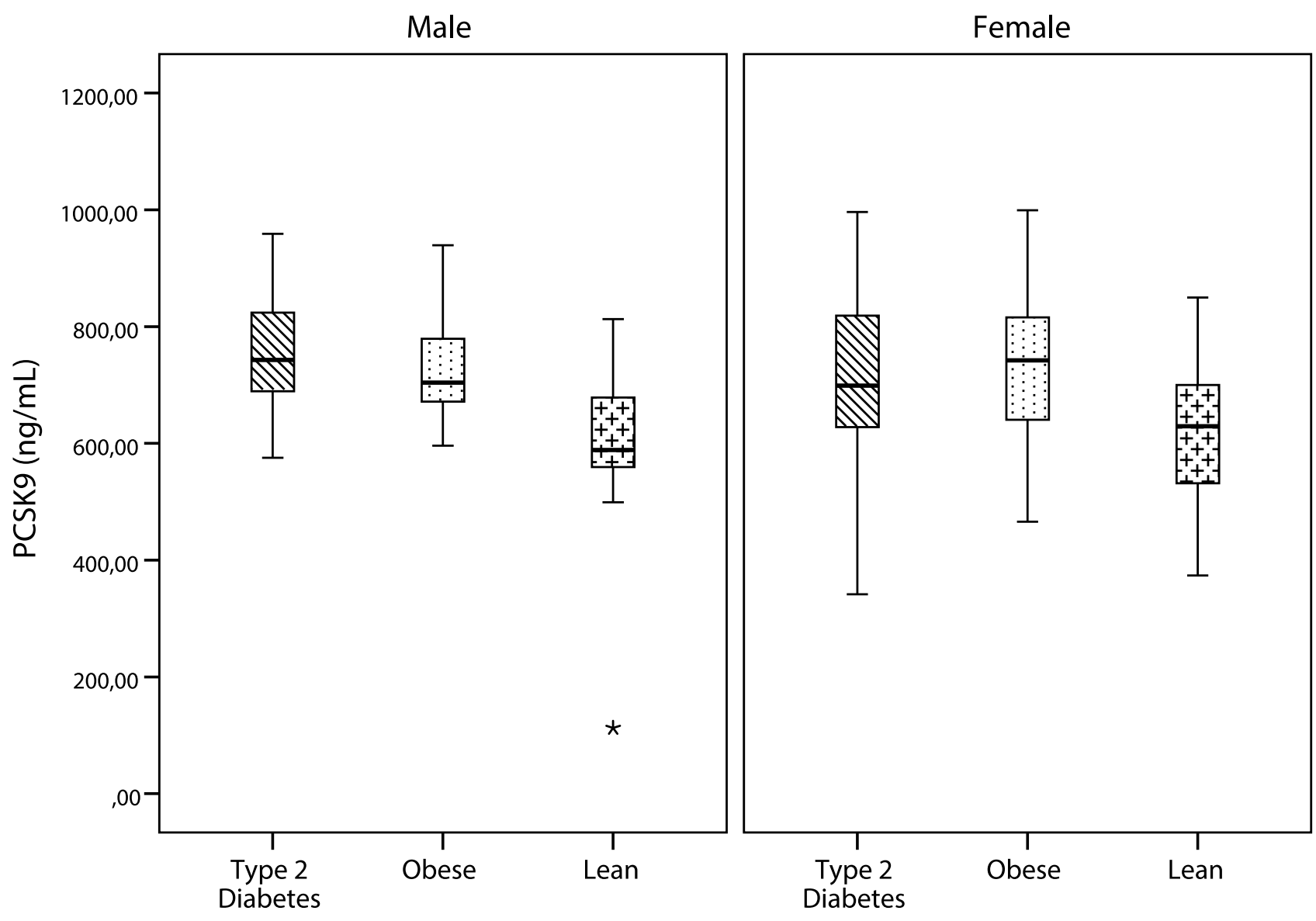

Group

Figure 2 Comparison of PCSK9 by group within each sex. PCSK9 levels remained significantly higher in patients with obesity and type 2 diabetes even after stratification by gender.

Consistent with pre-clinical studies, and observation studies, our study suggests that hyper-insulinemic in insulinresistant states like type 2 diabetes and obesity may influence PCSK9 levels. ${ }^{10}$ Similarly, Levenson et al, in a pediatric population showed PCSK9 levels to be positively correlated to insulin levels. ${ }^{16}$ So far, studies are inconsistent as Brower's et al did not find an association between PCSK9 and diabetes. ${ }^{14}$ Overall, studies that did not find an association between diabetes and PCSK9 were conducted mostly in Caucasian populations whereas studies that found a positive association between PCSK9 and diabetes were mainly conducted in ethnically diverse cohorts. $^{8}$ This suggests that the effects of diabetes on PCSK9 may be influenced by the race. Although PCSK9 concentrations did not differ significantly between the obese and type 2 diabetes groups, pre-clinical studies suggest the role of additional factors to insulin-like SREBP-1 and SREBP-2 and HNF-1 $\alpha$ in the induction of PCSK9 in patients with obesity and type 2 diabetes. ${ }^{10,11}$
Multivariate analysis showed that BMI was independently predictive for PCSK9 levels. This relationship was maintained after adjusting for potential confounders such as age, sex and HbA1c. Consistent with prior studies, we found this association of BMI with PCSK9 levels only present in females suggesting that gender might be an effect modifier in the relationship between BMI and PCSK9 levels. ${ }^{16}$

PCSK9 levels were positively correlated with triglyceride levels in the male group but not with LDL cholesterol. Plasma PCSK9 measured in ethnically diverse cohorts show that circulating PCSK9 levels vary widely among apparently healthy subjects and correlate differently with the plasma LDL concentration. ${ }^{8,12}$ It is thought that genetic factors contribute to this variability.

PCSK9 gene is thought to be one of the genes that regulate both cholesterol and glucose metabolism. ${ }^{12}$ We did not find a correlation between PCSK9 levels and glycemic control. Yang et al in a Chinese cohort found a positive correlation 
Table 3 Correlation of PCSK9 with Clinical Variables by Sex

\begin{tabular}{|l|l|l|l|l|}
\hline \multirow{2}{*}{ Clinical Variable } & \multicolumn{2}{l|}{ Females } & \multicolumn{2}{l|}{ Males } \\
\cline { 2 - 5 } & Correlation Coefficient & p-value & \multicolumn{1}{l|}{ Correlation Coefficient } \\
\hline Age (years) & -0.02 & 0.87 & 0.12 & 0.26 \\
Systolic BP $(\mathrm{mmHg})$ & 0.28 & 0.15 & 0.04 & 0.83 \\
Diastolic BP $(\mathrm{mmHg})$ & 0.43 & 0.85 & 0.21 & 0.30 \\
BMI $\left(\mathrm{Kg} / \mathrm{m}^{2}\right)$ & 0.61 & $\mathbf{0 . 0 4}$ & 0.05 & 0.63 \\
Fasting glycaemia $(\mathrm{g} / \mathrm{L})$ & 0.61 & 0.82 & 0.53 & 0.21 \\
Glycated hemoglobin $(\%)$ & 0.53 & 0.1 & 0.39 & 0.92 \\
Total cholesterol $(\mathrm{g} / \mathrm{L})$ & 0.09 & 0.43 & 0.05 & 0.67 \\
HDL cholesterol $(\mathrm{g} / \mathrm{L})$ & 0.17 & 0.12 & -0.13 & 0.23 \\
LDL cholesterol $(\mathrm{g} / \mathrm{L})$ & 0.06 & 0.56 & -0.03 & 0.77 \\
Triglycerides $(\mathrm{g} / \mathrm{L})$ & 0.15 & 0.17 & 0.56 & $\mathbf{0 . 0 2}$ \\
hs-CRP $(\mathrm{mg} / \mathrm{dL})$ & 0.51 & 0.39 & 0.17 & 0.09 \\
\hline
\end{tabular}

Notes: Boldface type indicates statistical significance where $\mathrm{p}<0.05$. PCSK9 levels were positively correlated to BMI in females and to triglyceride levels in males. Abbreviations: BMI, body mass index; BP, blood pressure; FPG, fasting plasma glucose; HbAlc, glycated hemoglobin; HDL, high-density lipoprotein; LDL, low-density lipoprotein; PCSK9, Proprotein convertase subtilisin/kexin type 9; hs-CRP, High sensitive C-reactive protein.

between PCSK9 levels and glycated hemoglobin in type 2 diabetes subjects but not in controls. ${ }^{17}$ Clinical trials with PCSK9 inhibitors report a small but significant increase in plasma glycaemia and $\mathrm{HbA1c}$, without increasing the risk of incident type 2 diabetes in the short term. ${ }^{12}$

Type 2 diabetes and obesity are often associated with dyslipidemia which increases cardiovascular risk. Our data show that mean serum triglyceride, LDL and total cholesterol levels were higher in type 2 diabetes subjects compared to lean subjects. Conversely, Yadav et al showed that lipid levels were increased in patients with T2D. ${ }^{18}$ This is because T2D is associated with increased deposition of triglycerides in nonadipose tissue, such as the heart, liver, pancreas, and skeletal muscle. Dyslipidemia in this population is characterized by hypertriglyceridemia and low levels of HDL-C. It is more common in patients with diabetes as compared to subjects without diabetes due to a fourfold increase in VLDL triglyceride. Lipid abnormalities in type 2 diabetes could be secondary consequences of insulin resistance; therefore, any approach that lowers insulin resistance would be anticipated to have a beneficial effect on dyslipidemia. ${ }^{3}$

PCSK9 is a cardiovascular risk factor that not only regulates cholesterol metabolism but acts as a critical regulator of inflammatory reaction. It has been shown to be positively correlated with hs-CRP which is also an independent cardiovascular disease (CVD) risk marker. ${ }^{7}$ Our data indicate that hsCRP levels differed significantly among the 3 groups but there was no correlation between PCSK9 and hs-CRP.

In summary, in an adult population of patients with obesity without diabetes, non-obese type 2 diabetes, and lean nondiabetic subjects, we showed that PCSK9 levels were significantly higher in the T2D and obese groups compared to the lean group. Women compared to men have a lower cardiovascular disease risk in the absence of diabetes and obesity. But in females with diabetes, the risk of CVD is similar to that of non-diabetic males; therefore, it is important to assess sex-dependent PCSK9 levels in these groups.

Some limitations of this study warrant attention. We did not assess menopausal status in the female subjects which is important because pre and post-menopausal statuses have been shown to influence PCSK9 levels. However, given the very few post-menopausal women in our sample, it is unlikely that differences in menopausal status confounded our results. Also, the hospital-based sampling limits generalizability.

\section{Conclusion}

Our results show higher PCSK9 levels in subjects with obesity and type 2 diabetes suggesting that these metabolic states potentially influence PCSK9. It is possible that in obese/type 2 diabetes subjects, PCSK9 plays a role in the increased cardiovascular outcomes risk, given that findings suggest that PCSK9 predicts future cardiovascular events. More work is needed to understand the contribution of high PCSK9 levels in obese subjects and patients with type 2 diabetes in this population.

\section{Ethical Consideration}

This study was approved by the Institutional Review Board and Regional Ethics committee. All participants provided written informed consent prior to inclusion. 


\section{Acknowledgments}

The authors acknowledge the contribution of Mr Djahmeni Eric and Mrs Tchiegang Christine for their assistance in the biochemical analysis of the samples at the laboratory of the National Obesity Center. We are grateful to the participants who took part in this study.

\section{Author Contributions}

All authors contributed to data analysis, drafting and revising the article, gave final approval of the version to be published, and agree to be accountable for all aspects of the work.

\section{Disclosure}

The authors declare no conflicts of interest in this work.

\section{References}

1. Cho NH, Shaw JE, Karuranga S, et al. IDF diabetes atlas: global estimates of diabetes prevalence for 2017 and projections for 2045. Diabetes Res Clin Pract. 2018;138:271-281. doi:10.1016/j.diabres.2018.02.023

2. Chehade JM, Gladysz M, Mooradian AD. Dyslipidemia in type 2 diabetes: prevalence, pathophysiology, and management. Drugs. 2013;73(4):327-339. doi:10.1007/s40265-013-0023-5

3. Klop B, Elte JWF, Castro Cabezas M. Dyslipidemia in obesity: mechanisms and potential targets. Nutrients. 2013;5(4):1218-1240. doi:10.3390/nu5041218

4. Jaiswal M, Schinske A, Pop-Busui R. Lipids and lipid management in diabetes. Best Pract Res Clin Endocrinol Metab. 2014;28(3):325-338. doi:10.1016/j.beem.2013.12.001

5. Schulz R, Schlüter K-D, Laufs U. Molecular and cellular function of the proprotein convertase subtilisin/kexin type 9 (PCSK9). Basic Res Cardiol. 2015;110(2):4. doi:10.1007/s00395-015-0463-z

6. Ruscica M, Tokgözoğlu L, Corsini A, Sirtori CR. PCSK9 inhibition and inflammation: a narrative review. Atherosclerosis. 2019;288:146-155. doi:10.1016/j.atherosclerosis.2019.07.015

7. Momtazi-Borojeni AA, Sabouri-Rad S, Gotto AM, et al. PCSK9 and inflammation: a review of experimental and clinical evidence. Eur Heart $J$ Cardiovasc Pharmacother. 2019;5(4):237-245. doi:10.1093/ehjcvp/ pvZ022
8. Lakoski SG, Lagace TA, Cohen JC, Horton JD, Hobbs HH. Genetic and metabolic determinants of plasma PCSK9 levels. $J$ Clin Endocrinol Metab. 2009;94(7):2537-2543. doi:10.1210/ jc.2009-0141

9. Caselli C, Del Turco S, Ragusa R, et al. Association of PCSK9 plasma levels with metabolic patterns and coronary atherosclerosis in patients with stable angina. Cardiovasc Diabetol. 2019;18(1):144. doi:10.1186/s12933-019-0949-3

10. Miao J, Manthena PV, Haas ME, et al. The role of insulin in the regulation of PCSK9. Arterioscler Thromb Vasc Biol. 2015;35 (7):1589-1596. doi:10.1161/ATVBAHA.115.305688

11. Dong B, Wu M, Li H, et al. Strong induction of PCSK9 gene expression through HNF1 $\alpha$ and SREBP2: mechanism for the resistance to LDLcholesterol lowering effect of statins in dyslipidemic hamsters. J Lipid Res. 2010;51(6):1486-1495. doi:10.1194/jlr.M003566

12. Schmidt AF, Swerdlow DI, Holmes MV, et al. PCSK9 genetic variants and risk of type 2 diabetes: a mendelian randomisation study. Lancet Diabetes Endocrinol. 2017;5(2):97-105. doi:10.1016/S22138587(16)30396-5

13. Saavedra YGL, Dufour R, Davignon J, Baass A. PCSK9 R46L, lower LDL, and cardiovascular disease risk in familial hypercholesterolemia: a cross-sectional cohort study. Arterioscler Thromb Vasc Biol. 2014;34(12):2700-2705. doi:10.1161/ ATVBAHA.114.304406

14. Brouwers MC, Troutt JS, Van Greevenbroek MM, et al. Plasma proprotein convertase subtilisin kexin type 9 is not altered in subjects with impaired glucose metabolism and type 2 diabetes mellitus, but its relationship with non-HDL cholesterol and apolipoprotein B may be modified by type 2 diabetes mellitus: the CODAM study. Atherosclerosis. 2011;217(1):263-267.

15. Whitley E, Ball J. Statistics review 4: sample size calculations. Crit Care London Engl. 2002;6(4):335-341. doi:10.1186/cc1521

16. Levenson AE, Shah AS, Khoury PR, et al. Obesity and type 2 diabetes are associated with elevated PCSK9 levels in young women. Pediatr Diabetes. 2017;4:2-8.

17. Yang S-H, Li S, Zhang Y, et al. Positive correlation of plasma PCSK9 levels with HbAlc in patients with type 2 diabetes. Diabetes Metab Res Rev. 2016;32(2):193-199. doi:10.1002/dmrr.v32.2

18. Yadav NK, Thanpari C, Shrewastwa MK, Mittal RK. Comparison of lipid profile in type-2 obese diabetics and obese non-diabetic individuals. a hospital based study from Western Nepal. Kathmandu Univ Med J. 2012;10(39):44-47. doi:10.3126/kumj. v10i3.8017
Diabetes, Metabolic Syndrome and Obesity: Targets and Therapy is an international, peer-reviewed open-access journal committed to the rapid publication of the latest laboratory and clinical findings in the fields of diabetes, metabolic syndrome and obesity research. Original research, review, case reports, hypothesis formation, expert opinion and commentaries are all considered for publication. The manuscript management system is completely online and includes a very quick and fair peer-review system, which is all easy to use. Visit http://www.dovepress.com/testimonials.php to read real quotes from published authors. 